\title{
The effects of hemorrhagic parenchymal infarction on the establishment of sensori-motor structural and functional connectivity in early infancy
}

\author{
T. Arichi • S. J. Counsell • A. G. Allievi • A. T. Chew • \\ M. Martinez-Biarge $\cdot$ V. Mondi $\cdot$ N. Tusor $\cdot$ N. Merchant $\cdot$ \\ E. Burdet • F. M. Cowan • A. D. Edwards
}

Received: 23 April 2014 / Accepted: 18 July 2014 / Published online: 14 August 2014

(C) The Author(s) 2014. This article is published with open access at Springerlink.com

\begin{abstract}
Introduction The objective of the study was to characterize alterations of structural and functional connectivity within the developing sensori-motor system in infants with focal perinatal brain injury and at high risk of cerebral palsy.

Methods Functional magnetic resonance imaging (fMRI) and diffusion tensor imaging (DTI) data were used to study the developing functional and structural connectivity framework in six infants born prematurely at term equivalent age. This was first characterised in three infants without focal pathology, which was then compared to that derived from three infants with unilateral haemorrhagic parenchymal infarction and a subsequent focal periventricular white matter lesion who developed later haemiparesis.

Results Functional responses to passive hand movement were in the contralateral perirolandic cortex, regardless of focal pathology. In infants with unilateral periventricular injury, afferent thalamo-cortical tracts appeared to have developed compensatory trajectories which circumvented areas of
\end{abstract}

Electronic supplementary material The online version of this article (doi:10.1007/s00234-014-1412-5) contains supplementary material, which is available to authorized users.

T. Arichi $(\bowtie) \cdot$ S. J. Counsell $\cdot$ A. T. Chew $\cdot$ V. Mondi $\cdot$ N. Tusor $\cdot$ N. Merchant $\cdot$ A. D. Edwards

Department of Perinatal Imaging \& Health, Division of Imaging Sciences \& Biomedical Engineering, Kings College London, St Thomas' Hospital, 1st floor North Wing, Westminster Bridge Road, London SE1 7EH, UK

e-mail: tomoki.arichi@kcl.ac.uk

T. Arichi $\cdot$ A. G. Allievi $\cdot$ E. Burdet $\cdot$ A. D. Edwards Department of Bioengineering, Imperial College London, London, UK

A. T. Chew $\cdot$ M. Martinez-Biarge $\cdot$ F. M. Cowan Department of Paediatrics, Imperial College Healthcare NHS Trust, London, UK damage. In contrast, efferent corticospinal tracts showed marked asymmetry at term equivalent age following focal brain injury. Sensori-motor network analysis suggested that inter-hemispheric functional connectivity is largely preserved despite pathology and that impairment may be associated with adverse neurodevelopmental outcome.

Conclusion Following focal perinatal brain injury, altered structural and functional connectivity is already present and can be characterized with MRI at term equivalent age. The results of this small case series suggest that these techniques may provide valuable new information about prognosis and the pathophysiology underlying cerebral palsy.

Keywords Neonatal · Focal brain lesion · fMRI · Diffusion MRI $\cdot$ Connectivity

In the third trimester of gestation, the brain's connectivity framework is established through a complex but highly programmed sequence of maturation, mediated through key transient developmental structures such as the subcortical subplate [1]. The importance of this period for life-long brain structure and function is emphasized by marked increases in the prevalence of neurodevelopmental impairment in survivors of preterm birth, in comparison to their peers born at full term [2]. Recent advances in imaging have now made it possible to non-invasively characterize the large-scale architecture of the brain's emerging pattern of functional and structural connectivity using blood oxygen level dependent (BOLD) functional magnetic resonance imaging (fMRI) and diffusion-weighted MRI [3]. Significantly, while such connectivity measures have been found to be altered in relation to clinical outcome in the mature brain, the specific effects of focal pathology on the establishment of intrinsic connectivity within the developing brain during early infancy have not previously been explored $[4,5]$. 
Preterm infants are at risk of intra-ventricular haemorrhage (IVH) and periventricular haemorrhagic parenchymal infarction (HPI) [6]. The developing sensori-motor system is particularly vulnerable as its pathways course through the commonest site of injury, with unilateral HPI typically resulting in cerebral palsy and a contralesional haemiparesis [6]. However, there have also been a number of case reports describing altered patterns of functional activity in later childhood following perinatal brain injury, which may reflect the newborn brain's increased capacity for neural plasticity and reorganization $[7,8]$. In this study, we aimed to characterize the specific effects of HPI on the developing sensori-motor system of infants with later cerebral palsy by precisely localizing functional responses to a passive motor task with fMRI, by delineating the afferent thalamo-cortical and efferent corticospinal tracts (CSTs) with diffusion tensor imaging (DTI) probabilistic tractography and by exploring intrinsic functional connectivity within a putative sensori-motor network. These techniques were first applied to provide a characterization of the sensori-motor connectivity framework in the absence of focal pathology in three 'control' prematurely born infants with normal motor outcome. They were then used to study three 'case' infants with unilateral HPI and a subsequent periventricular white matter lesion, who later developed contralateral spastic cerebral palsy. We hypothesized that in comparison to infants without focal brain injury, topological and quantitative differences in functional and structural connectivity would already be present at term equivalent age (TEA) in infants with HPI, which would be consolidated at 1 year of age.

\section{Method}

The work was approved by the NHS Research Ethics Committee, and written parental consent was obtained prior to all data acquisition. Data were collected at the Queen Charlotte and Chelsea Hospital, London, UK between 2009 and 2012.

\section{Participants}

The study population consisted of six prematurely born infants: three infants known not to have focal brain pathology (controls) (selected from a previously described cohort [9]) and three infants diagnosed on cranial ultrasound examination with unilateral HPI (cases) and a consequent focal periventricular white matter lesion. All six infants were studied using MRI at TEA, and case infants were additionally studied at 1 year of corrected age (see Table 1). All infants were seen at 1-year corrected age for a neurodevelopmental assessment using the Griffiths Mental Developmental Scale (revised) (GMDS-R) and an age-appropriate standardized neurological examination [10-12].
MR image acquisition

Infants were clinically assessed by a paediatrician, who was in attendance throughout the MRI scan. All infants were given chloral hydrate sedation $(30-50 \mathrm{mg} / \mathrm{kg}$ at TEA; $50-80 \mathrm{mg} / \mathrm{kg}$ at 1-year corrected age) $20 \mathrm{~min}$ prior to image acquisition. During scanning, temperature, oxygen saturations and heart rate were monitored, hearing protection was applied (dental putty and adhesive ear muffs (MiniMuffs, Natus Medical Inc., San Carlos, CA, USA), and the head was immobilized with a vacuum-evacuated polystyrene-bead-filled pillow [13].

MRI data were collected with a 3-T Philips Achieva MRI system (Best, the Netherlands), with an eight-channel phasedarray head coil. High-resolution conventional images were acquired using a turbo spin echo (TSE) T2-weighted sequence and a 3D magnetization-prepared rapid acquisition gradient echo (MP-RAGE) T1-weighted sequence and reviewed by a perinatal neuroradiologist [13]. fMRI data were collected with a single-shot gradient echo echo-planar imaging (EPI) sequence lasting $6 \mathrm{~min}$ and $34 \mathrm{~s}$ (parameters: in-plane resolution $2.5 \mathrm{~mm} * 2.5 \mathrm{~mm}$; 22 slices; slice thickness $3.25 \mathrm{~mm}$; repetition time (TR) 1,500 ms; echo time (TE) $45 \mathrm{~ms}$; flip angle (FA) $90^{\circ}$; total 256 volumes). DTI data were collected in 32 non-collinear directions with a $b$ value of $750 \mathrm{~s} / \mathrm{mm}^{2}$ using a spin echo EPI sequence (parameters: in-plane resolution $1.75 \mathrm{~mm} * 1.75 \mathrm{~mm}$; 49 slices; slice thickness $2 \mathrm{~mm}$; TR 9,000 ms; TE $49 \mathrm{~ms}$ ). Passive sensori-motor stimulation for the fMRI experiment was performed using a simple block paradigm (24 s of passive movement alternating with $24 \mathrm{~s}$ of rest) and a fully automated hand interface in the form of a custom-made inflatable balloon which was placed in the palm contralateral to the known periventricular lesion in case infants (or right hand in controls) [9]. Inflation of the balloon resulted in passive finger extension (and deflation in finger flexion), and the timing of stimulation was fully synchronized with fMRI data acquisition [9].

Image analysis

Image analysis was performed using tools implemented in the FMRIB Software Library (FSL, Oxford, UK) (http://fsl.fmrib. ox.ac.uk/fsl/fslwiki/) [14]. Standard preprocessing steps as implemented in FEAT v5.98 were performed on the fMRI data including motion correction, spatial smoothing (5-mm FWHM), highpass temporal filtering (cut-off $50 \mathrm{~s}$ ) and data denoising using MELODIC (Model-free FMRI analysis using probabilistic independent component analysis (PICA, v3.0)) [14]. Time-series statistical analysis was performed using the general linear model (GLM), with the measured data represented by a convolution of the experimental design and an age-appropriate basis set generated using FLOBS (FMRIB's linear optimal basis sets) $[14,15]$. Calculated parameter estimates were then converted to a $z$-statistical score image 


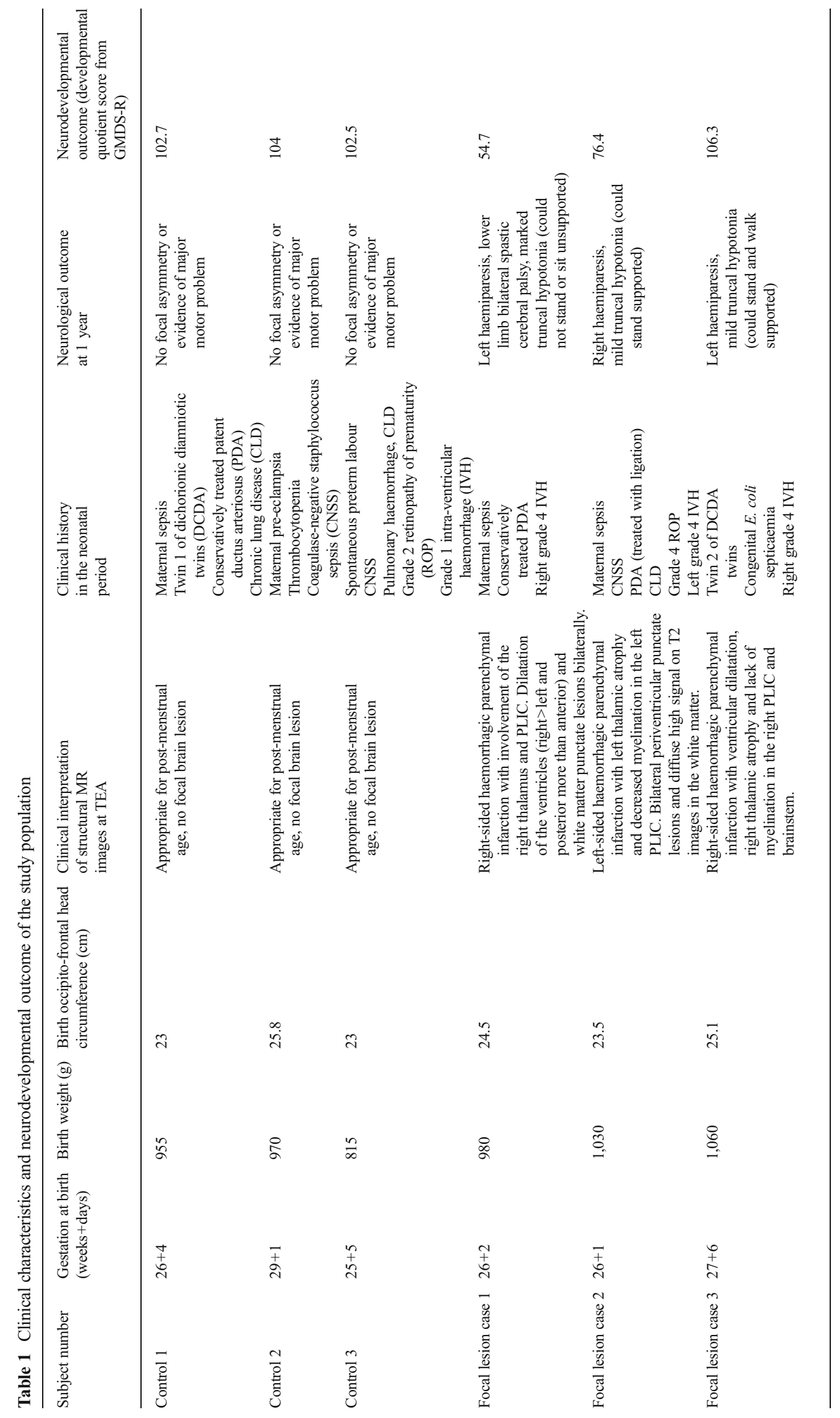


(threshold 2.3) with a corrected cluster significance level of $p<0.05$.

The afferent thalamo-cortical and efferent CSTs were delineated by probabilistic tractography using tools implemented in FSL's Diffusion Toolbox (FDT) [14]. Image volumes representing gradient directions corrupted by motion artefact during acquisition were excluded from the total data set [16]. Standard preprocessing steps were applied (non-brain tissue removal, eddy current correction), and fractional anisotropy (FA), radial diffusivity (RD), and axial diffusivity (AD) were calculated by fitting the data to the diffusion tensor model [17]. Voxelwise diffusion parameter distributions were then calculated using Bayesian Estimation of Diffusion Parameters Obtained using Sampling Techniques, X stands for modelling crossing fibres (BEDPOSTX) [14]. The thalamo-cortical tracts were then identified using a manually delineated seed in the thalamus ipsilateral to a waypoint mask derived from the top quartile of $z$ scores within the subject-specific fMRI activation cluster in the perirolandic region. This process was repeated for both hemispheres in infants with bilateral patterns of functional activity and for functional clusters in the insula where relevant. The CSTs were delineated separately using a set of anatomically defined regions (the cerebral peduncle, posterior limb of the internal capsule (PLIC) and perirolandic cortex) [18]. The identified connectivity distributions were then normalized by the number of samples passing from the seed mask through the distal waypoint areas and were thresholded at $1 \%$ [17]. For each of the identified CSTs, volume and diffusion metrics (FA, RD and $\mathrm{AD}$ ) were determined and an asymmetry index (AI) for these values calculated using the formula $[2 *$ (mean diffusion metric contralesional tract - mean diffusion metric ipsilesional tract)/(mean diffusion metric contralesional tract + mean diffusion metric ipsilesional tract)] [18]. For control infants, this formula was adapted to $[2 *($ mean left tract - mean right tract $) /($ mean left tract + mean right tract)] [18].

\section{Functional connectivity analysis}

Intrinsic functional connectivity was studied within a putative sensori-motor network consisting of nine anatomically defined $6-\mathrm{mm}$ radius sphere masks. These were placed in the bilateral perirolandic areas (encompassing the primary motor (M1) and somatosensory (S1) cortices), supplementary motor area (SMA) (a single mask that contained both left and right sides), bilateral insular areas (including the overlying opercular cortex and secondary somatosensory cortex S2), bilateral basal ganglia and bilateral thalami (see Supplementary Figure) [19]. To remove the effects of stimulus-induced signal change, only the residuals from the GLM analysis were used for this analysis, as it has been previously described to give a good representation of the underlying resting state signal [20].
Further band-pass temporal filtering was not performed in view of the risk of producing edge artefacts. The mean eigen-time series was extracted from each mask, and pairwise Pearson's partial correlation coefficients calculated using the Statistics Toolbox of MATLAB (2012a, the MathWorks, Natick, MA, USA). The six estimated head motion parameters (translations and rotations) and the cerebrospinal fluid (CSF) time series were included as confound regressors. A connectivity distribution map was then prepared using Cytoscape v3.0 (http://www.cytoscape.org/2 using only the significant connections as identified by a false discovery rate correction (FDR) of $p<0.05$ [21].

\section{Results}

Conventional MR imaging and neurodevelopmental outcome

Control infants had no focal brain lesions on conventional MR images, had no evidence of asymmetrical neurological signs and had a neurodevelopmental outcome within the normal range at 1-year corrected age (Table 1). All three cases at TEA and 1-year corrected age had a large periventricular white matter lesion at the site of the HPI, with suboptimal myelination in the PLIC and decreased thalamic volume ipsilateral to the cyst (in comparison with the non-lesional side), and the appearances were therefore highly predictive of later unilateral spastic cerebral palsy [22]. Although all case infants had a clear area of unilateral periventricular white matter injury (as a result of the HPI), there were also differing degrees of ventriculomegaly and global white matter abnormality, with case 1 the most severely affected with appearances consistent with periventricular leukomalacia (PVL). At 1year corrected age, all cases had asymmetrical neurological signs consistent with unilateral spastic cerebral palsy. This was most severe in case 1, who also had marked global neurodevelopmental delay (overall developmental quotient (DQ) score, 54.7). In contrast, while case 3 also had asymmetrical neurological signs, neurodevelopmental assessment was within the normal range (DQ score, 106.3).

\section{Functional imaging}

In all infants at TEA, positive BOLD functional clusters were identified in the perirolandic region contralateral to the side of passive sensori-motor stimulation (Figs. 1 and 2). In control infants, responses were localized to the 'hand' area of the contralateral perirolandic cortex with additional clusters in the SMA and were bilateral in one infant. In case infants, responses were seen bilaterally at TEA in cases 1 and 3 but only in the lesioned hemisphere in case 2. Functional clusters 


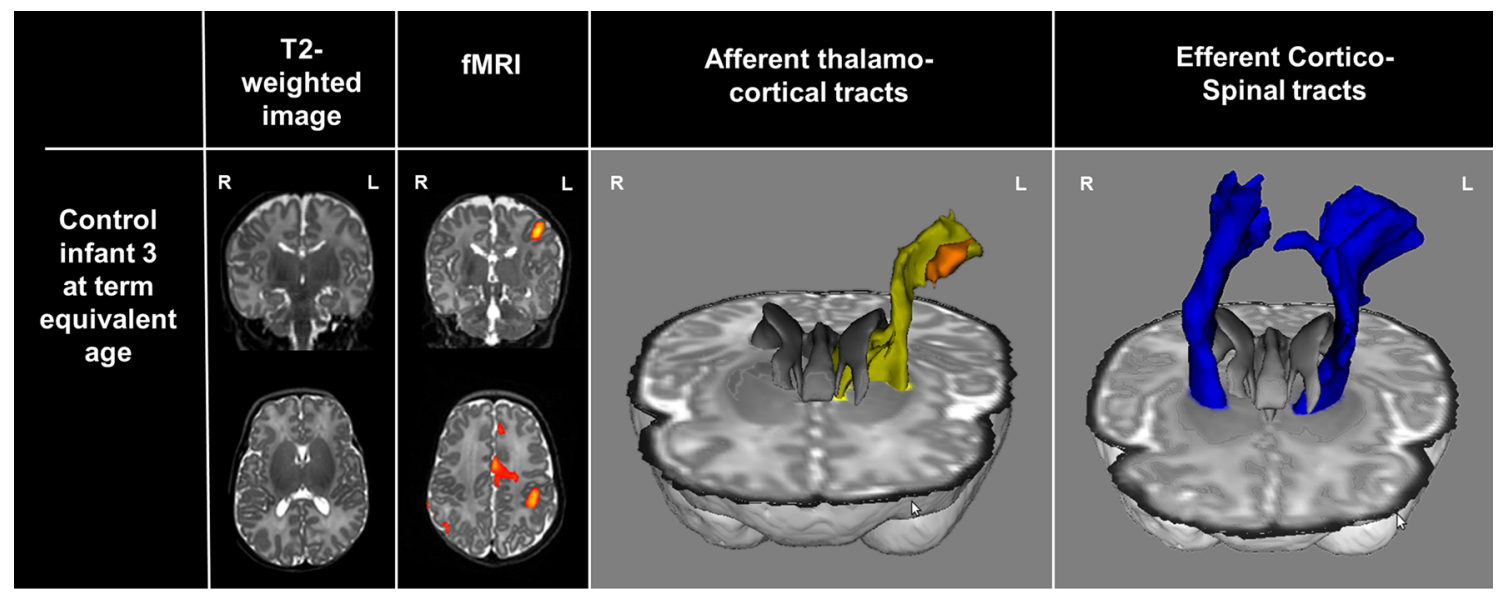

Fig. 1 Functional activation and probabilistic tractography in a control preterm infant without focal brain injury and imaged at term equivalent age. Following passive sensori-motor stimulation of the right hand, clusters of functional activity were identified in the contralateral (left) perirolandic region and supplementary motor area ( $z$-score threshold 2.3).

were also displaced within the perirolandic cortex posteriorly (case 2 ) or inferiorly and laterally (case 3 ). Activity was not identified in the SMA in case infants, although additional clusters were seen inferiorly in the insula in case 1.

At 1-year corrected age, functional responses remained in the lesioned hemisphere in case infants (Fig. 2). Functional activity in case 1 was seen exclusively in the perirolandic region of the lesional hemisphere (having previously had bilateral S1 responses) with additional areas in the SMA, insula and parietal operculum. Cases 2 and 3 retained a similar pattern to that seen at TEA, with unilateral and bilateral responses, respectively.

\section{DTI tractography of the thalamo-cortical tracts}

At TEA, afferent thalamo-cortical tracts could be delineated in all six infants from the thalamus running supero-laterally to the fMRI cluster in the perirolandic cortex contralateral to the side of stimulation (Figs. 1 and 2). In cases 2 and 3, the tracts had developed an altered trajectory which circumvented the area of focal periventricular white matter injury through additional curvature at both TEA and 1 year of age. In case 1 at TEA, it was not possible to delineate a tract running directly from the thalamus to the perirolandic region, although this connection was found to be possible through an indirect route of two tracts, the first of which connected the thalamus to the insula area (using the identified functional cluster in this region as a target for the tractography) and the second then connecting the insula superiorly to the area of functional activity in the perirolandic region. A single thalamo-cortical pathway could then be delineated at 1 year of age running through the insula (and passing through the functional
The afferent thalamo-cortical tract (yellow) was identified using probabilistic tractography and the fMRI cluster (orange) as a target mask. Symmetrical efferent corticospinal tracts (blue) were identified using anatomical regions of interest

cluster still present in the area), suggesting possible consolidation of the two pathways into a single tract.

\section{DTI tractography of the corticospinal tracts}

In each of three control infants at TEA, there was no significant asymmetry between the left and right CSTs in either volume or diffusion metrics (Fig. 3). Bilateral CSTs were also identified in all three case infants at both TEA and 1 year of age, although with marked asymmetry in volume due to a reduction in the lesional hemisphere in comparison to the intact side (Figs. 2 and 3). At TEA, marked asymmetry was also seen in FA (reduced in the lesional hemisphere) and RD (increased in the lesional hemisphere) suggesting reduced microstructural integrity (Fig. 3). Despite their clinical outcome, consistent asymmetry between the CSTs in any of the diffusion metrics was not sustained at 1 year.

Functional connectivity of the sensori-motor network

Functional connectivity analysis revealed significant interhemispheric connectivity between the left and right insular/ opercular and perirolandic regions in all control infants, with additional bilateral connectivity to the SMA (Fig. 4). Consistent functional connectivity was also seen between the thalami and adjacent basal ganglia and bilaterally between the two thalami. Similarly in case infants, inter-hemispheric functional connectivity was largely preserved between the perirolandic and insular/opercular regions at both timepoints. At TEA, connectivity was not seen between the SMA and perirolandic area in the lesioned hemisphere, in contrast to the non-lesioned side. The exception was case 1, who had marked sensori-motor network 


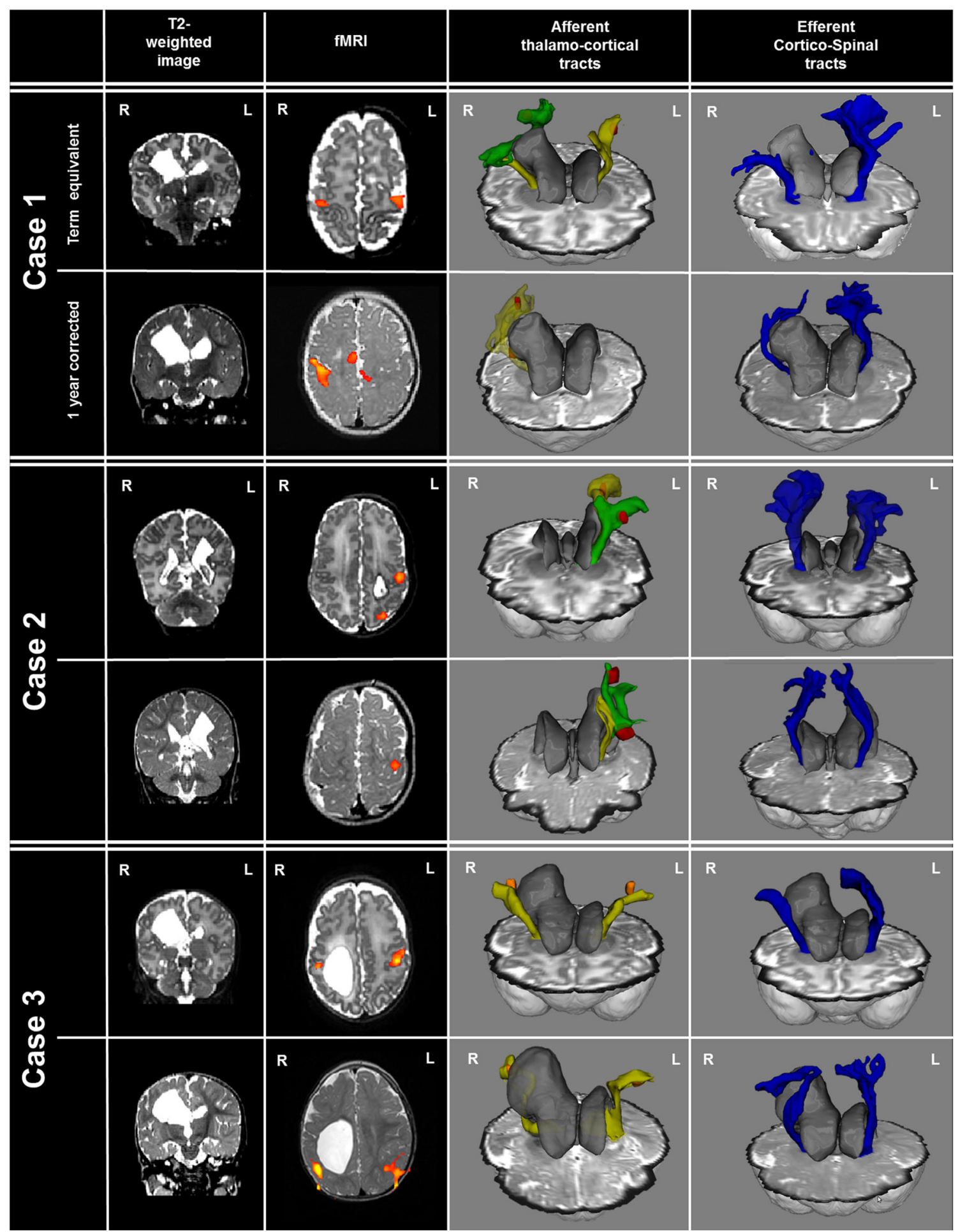

Fig. 2 Functional activation and probabilistic tractography in three cases with focal periventricular brain injury, studied at term equivalent and 1year corrected age. A unilateral periventricular white matter lesion can be seen arising from the lateral ventricle at the site of the previous haemorrhagic infarction on the right (cases 1 and 3) and left (case 2) sides. Following passive sensori-motor stimulation of the contralesional hand, clusters of functional activity were identified in all infants at both

disruption at both time-points, including a loss of functional connectivity between the perirolandic regions and time-points in the ipsilesional perirolandic region (z-score threshold 2.3). The afferent thalamo-cortical tracts (yellow and green) developed altered trajectories which circumvented the periventricular white matter lesion to meet the identified fMRI clusters (orange/red). Efferent corticospinal tracts (blue) showed marked asymmetry, with a decreased volume in the lesional hemisphere evident at both time-points

complete functional 'disconnection' of the ipsilesional basal ganglia at 1 year. 

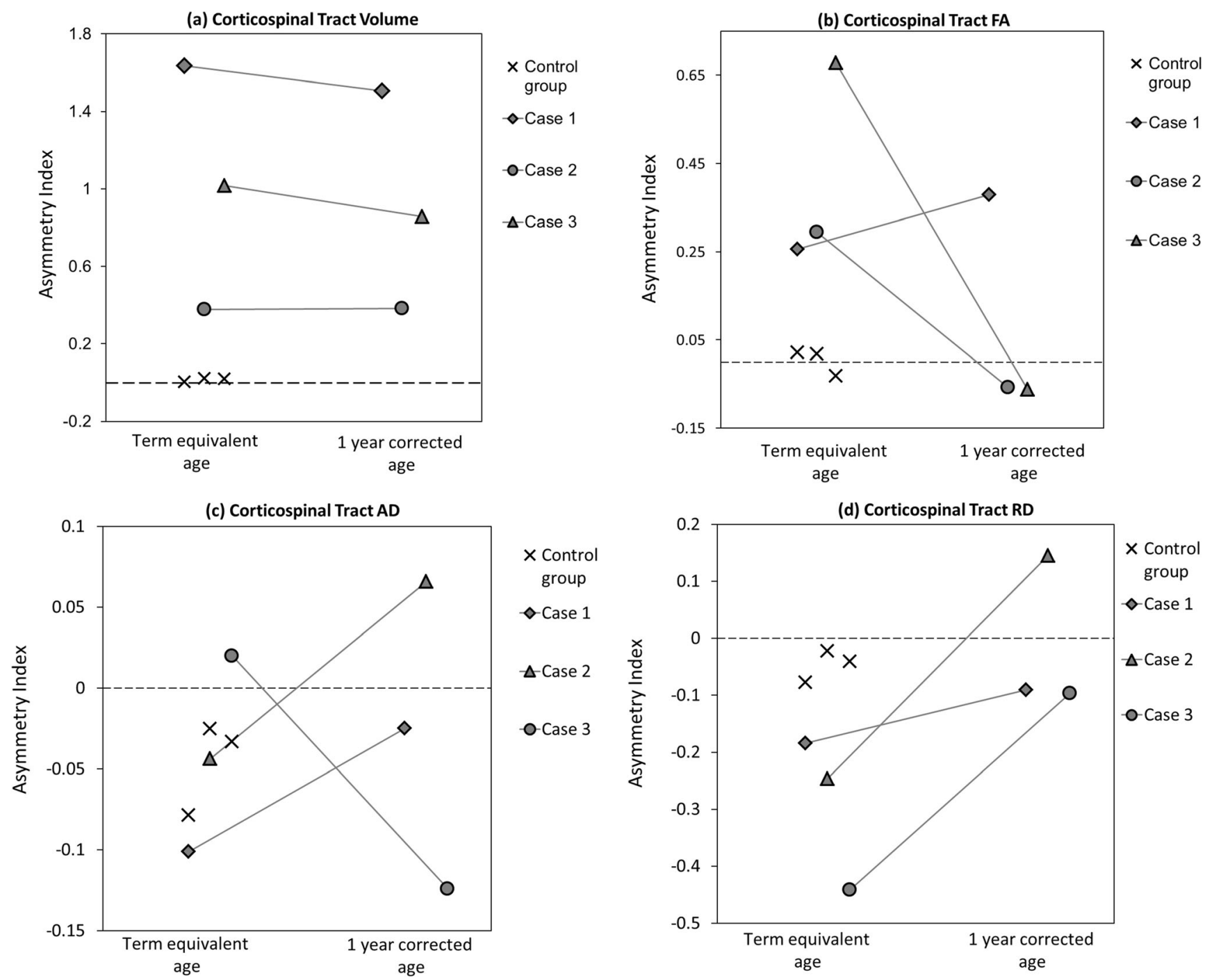

Fig. 3 Corticospinal tract volume and microstructural integrity. a In comparison to control infants (crosses), corticospinal volume in case infants was clearly asymmetrical at both term equivalent and 1-year corrected age. b, d Markers of microstructural integrity (fractional

\section{Discussion}

In infants who have suffered unilateral HPI and developed unilateral spastic cerebral palsy, alterations in the emerging architecture of sensori-motor functional and structural connectivity can be characterized in early infancy using advanced MRI techniques. We describe the normal framework of connectivity at TEA and provide evidence which suggests that differences due to perinatal brain injury are already present at TEA and precede the manifestation of later neurological and neurodevelopmental deficits. Regardless of focal brain pathology, functional responses were consistently seen in the perirolandic region contralateral to the side of passive sensori-motor stimulation, with the thalamo-cortical tracts appearing to have developed compensatory trajectories which circumvented areas of damage to reach the identified

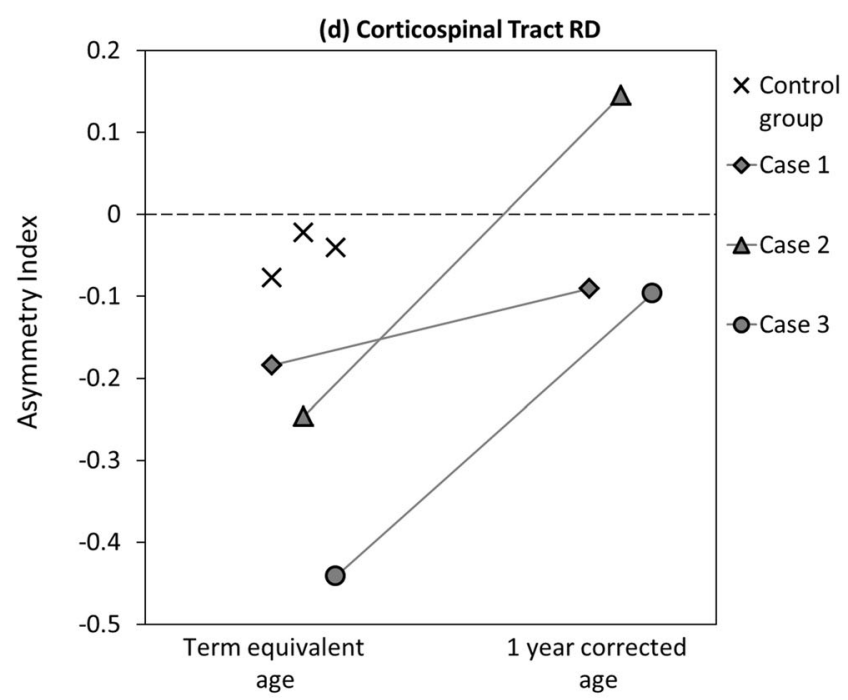

anisotropy $(F A)$ and radial diffusivity $(R D))$ were also asymmetric at term equivalent age in cases, although this was not sustained at 1 year of age. $\mathbf{c}$ There was no difference in asymmetry in axial diffusivity $(A D)$ at either time-point

functional activity. In contrast, marked asymmetry in the volume and microstructure of the efferent CSTs was seen at TEA in the presence of HPI. Functional connectivity analysis suggests that homotopic inter-hemispheric connectivity is integral to the sensori-motor network in early infancy and is largely preserved even following focal brain injury.

Our findings are in agreement with studies of older children and adolescents with similar patterns of periventricular brain injury, in which contralateral somatosensory functional responses to passive sensori-motor stimulation were also consistently seen $[23,24]$. In these cases, reorganization of somatosensory responses to the ipsilateral cortex appears to be rare and is associated with abnormal sensory function [24]. While we saw bilateral functional responses in two of the cases at TEA (and one of the controls), they are also observed in $40-60 \%$ of healthy infants and are thought to occur through 


\section{Case 1}
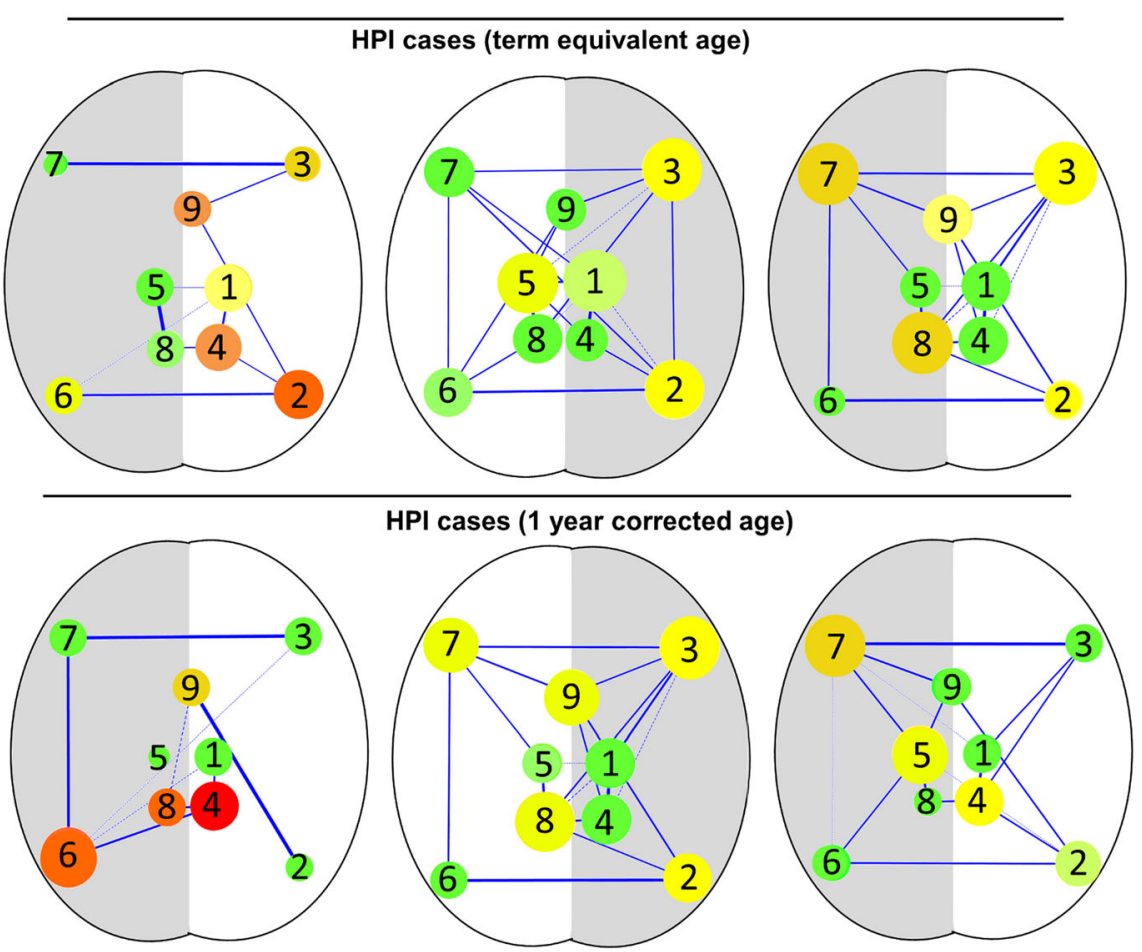

HPI cases (1 year corrected age)

0
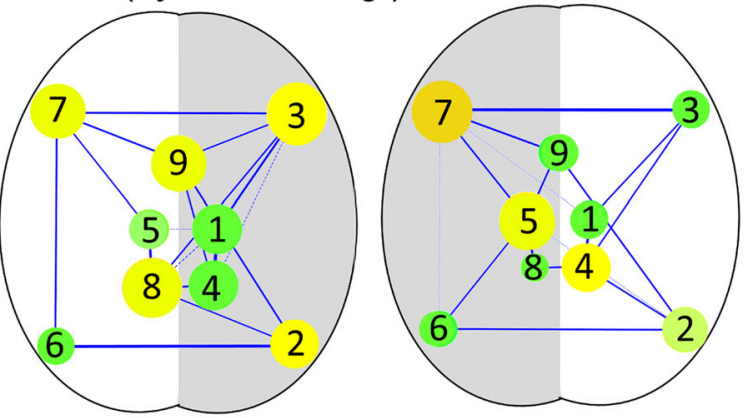

0.66

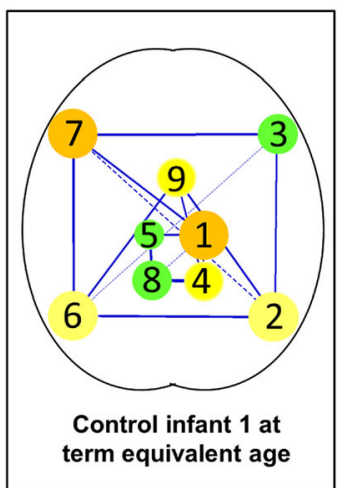

1. Left Basal Ganglia

2. Left peri-rolandic area

3. Left Insula and operculum

4. Left thalamus

5. Right Basal Ganglia

6. Right peri-rolandic area

7. Right insula and operculum

8. Right thalamus

9. Left and right Supplementary Motor Area

\section{Betweenness-centrality}

Fig. 4 Functional connectivity in the sensori-motor network. In an example control infant (inset box), a clear pattern of functional connectivity can be seen between one brain region and its homotopic counterpart in the opposite hemisphere. In case infants, functional connectivity between the lesional (grey) and non-lesional hemisphere (white) is largely preserved even in the presence of focal brain pathology. The exception is case 1 at 1 year of age, who has lost inter-hemispheric connectivity between the perirolandic regions and has functionally 'disconnected' ipsilesional basal ganglia. Intra-hemispheric connectivity between the perirolandic regions and the supplementary motor area (SMA) is absent in the lesional hemisphere in all cases at term equivalent age and the majority at 1 year of age. Node sizes are scaled by degree (the number of connected edges), node colour is scaled by betweeness centrality (a measure of the amount of control that a node exerts over the interactions of other nodes in the network), and edge thickness is scaled by the pairwise Pearson's partial correlation coefficient. Only edges which survived a false discovery rate correction (FDR) correction of $p<0.05$ are shown

compensatory trajectories following injury acquired during this period, in marked contrast to the growing afferent thalamo-cortical tracts $[8,23,25]$. Asymmetry in CST volume in the neonatal period was sustained at 1 year of corrected age, which may represent impaired development of the ipsilesional fibres or possible hypertrophy of the contralesional fibres (or a combination of both) $[8,25]$. While asymmetry of the CST was also observed in markers of microstructural integrity (FA and RD) at TEA, we did not find this at 1-year corrected age despite a clear functional manifestation (haemiparesis). This discrepancy may represent limitations inherent to the diffusion tensor model which struggles to model white matter microstructure in areas with inherently low anisotropy such as those containing crossing fibres [26]. Furthermore, as it was not possible to assess the functional activity of the primary motor cortex in these neonatal subjects, the tractography analysis of the CSTs could not be guided by the identified functional activity in the same way as the thalamo-cortical pathways. This would have been of great interest, as haemiparesis is 
often associated with consolidation of the ipsilateral CST from the healthy hemisphere to the paralyzed limb $[8,25]$. Further studies of both the afferent and efferent pathways could therefore benefit greatly from additional data derived through other functional imaging techniques (such as magnetoencephalography (MEG), electro-encephalography (EEG), or transcranial magnetic stimulation (TMS)) which can offer complementary information about pathway function with greater temporal specificity.

Sensori-motor network analysis revealed intact interhemispheric functional connectivity between the perirolandic regions in early infancy even in the presence of large brain lesions. This relationship was impaired only in case 1 at 1 year, which was notable given their poor neurodevelopmental outcome. In contrast, bilateral functional sensori-motor responses and preserved inter-hemispheric perirolandic connectivity were seen in case 3 at both time-points, who had the best neurodevelopmental outcome of the cases. Taken together, these results suggest that in the presence of a focal pathology and impaired CST development, preserved inter-hemispheric functional connectivity may be vital for positive neurodevelopmental outcome. Furthermore, this finding is in keeping with those of animal studies of hypoxic injury and in children following traumatic brain injury, which also observed a significant relationship between transcallosal connectivity and later neurobehavioural outcome [27, 28]. Of additional interest, we also found that intra-hemispheric functional connectivity between the perirolandic cortices and the SMA was impaired at TEA in the lesional hemisphere. The SMA is functionally active even during early infancy, and functional responses are consistently elicited with sensori-motor tasks [9, 15]. It has an important role in adult post-stroke recovery, with acute-phase 'hyper'-connectivity correlating with good motor recovery and increased activity seen during voluntary tasks chronically $[4,5]$.

Our work is limited by the small sample size and restricted time-points of assessment, and further longitudinal work will therefore be vital to build on our findings. These limitations are also pertinent given that all diffusion tractrography algorithms are susceptible to the identification of false positive and negative tracts (particularly at a single subject level) and given that MR-derived markers of white matter microstructural integrity are known to change during normal human development in addition to following injury [26]. To provide a precise characterization of the specific effects of a focal brain lesion, here, we intentionally studied only infants with similar injury in terms of location and timing, although further work is needed to study the effects of different perinatal lesions as they have been found to result in marked differences in neuroanatomy and functional outcome in later childhood [8, 23]. Given our specific study group, the effects of HPI on functional connectivity were explored only in the sensorimotor network in the context of their later cerebral palsy, although it would certainly be of interest to study the wider effects on other functional networks. We were also unable to fully explore some of the key relationships in the network due to constraints imposed by EPI acquisition sequences, which made it necessary to include more than one area (such as M1 and S1) in single masks and exclude other important structures (the cerebellum) entirely from our analysis. Future work would therefore also benefit greatly from acquiring diffusion-weighted data which would allow an improved estimation of white matter fibre orientation over the limited diffusion tensor model used here, such as what can be achieved with high-angular-resolution diffusion-weighted imaging (HARDI) [26]. Finally, in order to fully elucidate the functional role of the identified afferent and efferent pathways, it will also be necessary to collect higher temporal resolution data about the amplitude and morphology of the electrophysiological evoked responses within the pathological hemisphere using techniques such as EEG or MEG.

\section{Conclusions}

In summary, in this study, we demonstrate the feasibility of applying fMRI and diffusion tractography to offer new insights into the early effects of acquired perinatal brain injury in a small case series of infants with a clearly defined pattern of injury. We found that at TEA, functional responses to passive sensori-motor stimulation are in the contralateral perirolandic cortex, with established thalamo-cortical and symmetrical CST structural connectivity and a consistent pattern of interhemispheric functional connectivity. In the presence of unilateral HPI, passive sensori-motor functional responses remain in the lesional hemisphere, with an apparently altered but intact pattern of thalamo-cortical structural connectivity. In contrast, unilateral deficit of CST integrity at TEA is predictive of later contralateral motor impairment, and altered interhemispheric functional connectivity is associated with poor neurodevelopmental outcome. This small data series suggests that MRI in early infancy has a marked potential to provide new information regarding the pathophysiology of cerebral palsy, could be used as a possible biomarker for testing future therapeutic interventions and may provide new clinical prognostic information.

Ethical standards and patient consent We declare that all human and animal studies have been approved by the NHS Research Ethics Committee UK (West London), and have therefore been performed in accordance with the ethical standards laid down in the 1964 Declaration of Helsinki and its later amendments. We declare that all patients gave informed consent prior to inclusion in this study.

Acknowledgments TA and AGA received Fellowships from the Medical Research Council (MRC) UK and Engineering and Physical Sciences Research Council (EPSRC) UK, respectively. The work was supported 
by funding from the NIHR comprehensive Biomedical Research Centres (BRC) at Imperial College London and King's College London. The authors thank Professor Mary Rutherford, Professor Christian Beckmann and Mrs Joanna Allsop for comments and discussion throughout the work. The authors also thank the patients and families who participated in this work.

Conflict of interest We declare that we have no conflict of interest.

Open Access This article is distributed under the terms of the Creative Commons Attribution License which permits any use, distribution, and reproduction in any medium, provided the original author(s) and the source are credited.

\section{References}

1. Kostović I, Jovanov-Miloosević N (2006) The development of cerebral connections during the first 20-45 weeks' gestation. Semin Fetal Neonatal Med 11:415-422

2. Larroque B, Ancel PY, Marret S, Marchand L, André M, Arnaud C et al (2008) Neurodevelopmental disabilities and special care of 5year-old children born before 33 weeks gestation (the EPIPAGE study): a longitudinal cohort study. Lancet 371:813-820

3. Van Essen DC, Ugurbil K (2012) The future of the human connectome. Neuroimage 62:1299-1310

4. Carter AR, Shulman GL, Corbetta M (2012) Why use a connectivitybased approach to study stroke and recovery of function? Neuroimage 62:2271-2280

5. Grefkes C, Nowak DA, Eickhoff SB, Dafotakis M, Kust J, Karbe H et al (2008) Cortical connectivity after subcortical stroke assessed with functional magnetic resonance imaging. Ann Neurol 63:236-246

6. de Vries LS, Roelants-van Rijn AM, Rademaker KJ, Van Haastert IC, Beek FJ, Groenendaal F (2001) Unilateral parenchymal haemorrhagic infarction in the preterm infant. Eur J Pediatr Neurol 5:139-149

7. Fair DA, Brown TT, Petersen SE, Schlagger BL (2006) fMRI reveals novel functional neuroanatomy in a child with perinatal stroke. Neurology 67:2246-2249

8. Staudt M (2010) Brain plasticity following early life brain injury: insights from neuroimaging. Semin Perinatol 34:87-92

9. Arichi T, Moraux A, Melendez A, Doria V, Groppo M, Merchant N et al (2010) Somatosensory cortical activation identified by functional MRI in preterm and term infants. Neuroimage 49:2063-2071

10. Huntley M (1996) The Griffiths Mental Developmental Scales manual from birth to two years. Bucks: Association for Research in Infant and Child Development, The Test Agency, Thames; 5-39.

11. Mercuri E, Ricci D, Pane M, Baranello G (2005) The neurological examination of the newborn baby. Early Hum Dev 81:947-956

12. Haataja L, Mercuri E, Regev R, Cowan F, Rutherford M, Dubowitz V, Dubowitz L (1999) Optimality score for the neurologic examination of the infant at 12 and 18 months of age. J Pediatr 135:153-161

13. Merchant N, Groves A, Larkman DJ, Counsell SJ, Thomson MA, Doria V et al (2009) A patient care system for early 3.0 Tesla magnetic resonance imaging of very low birth weight infants. Early Hum Dev 85:779-783

14. Smith SM, Jenkinson M, Woolrich MW, Beckmann CF, Behrens TE, Johansen-Berg $\mathrm{H}$ et al (2004) Advances in functional and structural MR image analysis and implementation as FSL. Neuroimage 23: s208-s219

15. Arichi T, Fagiolo G, Varela M, Melendez-Calderon A, Allievi A, Merchant N et al (2012) Development of BOLD signal hemodynamic responses in the human brain. Neuroimage 63:663-673

16. Müller HP, Süssmuth SD, Landwehrmeyer GB, Ludolph A, Tabrizi SJ, Kloppel S et al (2011) Stability effects on results of diffusion tensor imaging analysis by reduction of the number of gradients due to motion artifacts: an application to presymptomatic Huntington's disease. PLoS Curr 3:RRN1292

17. Johansen-Berg H, Behrens TE (eds) (2009) Diffusion MRI: from quantitative measurement to in-vivo neuroanatomy. Academic Press, London, pp 333-353

18. Roze E, Harris PA, Ball G, Elorza LZ, Braga RM, Allsop JM et al (2012) Tractography of the corticospinal tracts in infants with focal perinatal injury: comparison with normal controls and to motor development. Neuroradiology 54:507-516

19. Mostofsky SH, Powell SK, Simmonds DJ, Goldberg MC, Caffo B, Pekar JJ (2009) Decreased connectivity and cerebellar activity in autism during motor task performance. Brain 132:2413-2425

20. Fair DA, Schlaggar BL, Cohen AL, Miezin FM, Dosenback NUF, Wenger KK et al (2007) A method for using block and event-related fMRI data to study "resting state" functional connectivity. Neuroimage 35:396-405

21. Shannon P, Markiel A, Ozier O, Baliga NS, Wang JT, Ramage D et al (2003) Cytoscape: a software environment for integrated models of biomolecular interaction networks. Genome Res 13:2498-2504

22. Boardman JP, Ganesan V, Rutherford MA, Saunders DE, Mercuri E, Cowan FM (2005) Magnetic resonance image correlated of hemiparesis after neonatal and childhood middle cerebral artery stroke. Pediatrics 115:321-326

23. Staudt M, Braun C, Gerloff C, Erb M, Grodd W, Krägeloh-Mann I (2006) Developing somatosensory projections bypass periventricular brain lesions. Neurology 67:522-525

24. Juenger H, de Haan B, Krägeloh-Mann I, Staudt M, Karnath HO (2011) Early determination of somatosensory cortex in the human brain. Cereb Cortex 21:1827-1831

25. Eyre JA, Smith M, Dabydeen L, Clowry GJ, Peyacchi E, Battini R et al (2007) Is hemiplegic cerebral palsy equivalent to amblyopia of the corticospinal system? Ann Neurol 62:493-503

26. Farquharson S, Tournier JS, Calamante F, Fabinyi G, SchneiderKolsky M, Jackson GD et al (2013) White matter fiber tractography: why we need to move beyond DTI. J Neurosurg 118:1367-1377

27. van Velthoven CTJ, van de Looij Y, Kavelaars A, Zijlstra J, van Bel F et al (2012) Mesenchymal stem cells restore cortical rewiring after neonatal ischemia in mice. Ann Neurol 71:785-796

28. Ewings-Cobbs L, Prasad MR, Swank P, Kramer L, Cox CS Jr et al (2008) Arrested development and disrupted callosal microstructure following pediatric traumatic brain injury: relation to neurobehavioral outcome. Neuroimage 42:1305-1315 